\title{
REGISTRO DA OCORRÊNCIA DE Meloidogyne incognita NO PORTA-ENXERTO 'IAC 766-CAMPINAS' NO ESTADO DE PERNAMBUCO E REAÇÃO DE PORTA-ENXERTOS E DE CULTIVARES COPA DE VIDEIRA A Meloidogyne spp. ${ }^{1}$
}

\author{
LÚCIA SOMAVILLA², CESAR BAUER GOMES³ ${ }^{2}$ VERA MARIA QUECINI ${ }^{4}$
}

RESUMO - Relata-se a ocorrência de Meloidogyne incognita (Est. I2) em pomar de videira na cidade de Petrolina, Pernambuco. Plantas da cultivar Festival enxertadas sobre 'IAC 766-Campinas', atacadas por Meloidogyne incognita, apresentavam sintomas de menor vigor, folhas amarelecidas e folhagem mais esparsa, e raízes com numerosas galhas em meio ao cultivo de feijão-de-porco (Canavalia ensiformis), também atacado pelo nematoide. Com o objetivo de avaliar a resistência de quatro porta-enxertos tropicais, oito de clima temperado, e as cultivares Niágara Rosada e Chardonnay quanto à reação a $M$. incognita e M. arenaria, em casa de vegetação, realizou-se este trabalho. Mudas das videiras, mantidas em vaso com solo esterilizado, foram inoculadas com 10.000 ovos + J2 de M. incognita ou M. arenaria por planta. Decorridos oito meses da inoculação, cada planta foi avaliada quanto ao número de galhas e número de ovos por planta, determinandose, a seguir, o fator de reprodução de cada espécie do nematoide (FR=população inicial/população final) nos diferentes materiais testados. Entre os porta-enxertos avaliados, 'Harmony’, 'Salt Creek', ‘1103 Paulsen’, 'IAC 572-Jales’, 'IAC 313-Tropical’, ‘K5BB Kober’ e 'SO4', foram resistentes (FR<1,00) e 'Solferino’, imune $(\mathrm{FR}=0,00)$ a $M$. incognita, sendo os demais suscetíveis ao nematoide. Quando se avaliou a reação da videira a M. arenaria, exceto '106-Traviú', ‘420 A', 'Rupestris du Lot' e '1103 Paulsen', os demais portaenxertos foram resistentes (FR>1,00) ao nematoide. No entanto, ambas as cultivares copa foram suscetíveis às duas espécies de Meloidogyne testadas.

Termos para indexação: Ocorrência, resistência, nematoide das galhas, Vitis spp.

\section{Meloidogyne incognita REPORT ON 'IAC 766-CAMPINAS' ROOTSTOCK IN THE STATE OF PERNAMBUCO AND REACTION OF GRAPE ROOTSTOCKS AND CULTIVARS TO Meloidogyne spp.}

\begin{abstract}
The occurrence of root-knot nematode Meloidogyne incognita (Est. I2) is reported in grapevine in Petrolina municipality, State of Pernambuco. Grape plants of Festival cultivar grafted on the 'IAC 766 Campinas' rootstock attacked by $M$. incognita exhibited symptoms of less vigorous growth, yellowing and sparse leaves and multiple galls in the root system in an area cultivated with jack-bean (Canavalia ensiformis) also infected by the same nematode. In order to evaluate resistance of four tropical rootstocks, eight temperate and two cultivars Chardonnay and Niagara Rosada in reaction to $M$. incognita and M. arenaria, this study was carried out. Seedlings of the investigated grapevine genotypes were maintained in pots with sterilized soil and subsequently inoculated with 10,000 eggs + J2 of $M$. incognita or $M$. arenaria per plant in a six replicate design. Eight months after nematode inoculation, each plant was evaluated by counting gall and eggs. The final number of eggs (FP) of each Meloidogyne specie was calculated and the reproductive factor $(\mathrm{RF}=\mathrm{FP} /$ IP), determined. Among the tested rootstocks, 'Harmony’, 'Salt Creek', ‘1103 Paulsen', 'IAC 572-Jales', 'IAC 313-Tropical', 'K5BB Kober', 'SO4', were resistant $(\mathrm{RF}<1.00)$ and, 'Solferino’, immune $(\mathrm{RF}=0.00)$ to $M$. incognita, the remaining rootstocks were susceptible to the nematode. With exception of '106-Traviú', '420 A', 'Rupestris du Lot' and '1103 Paulsen', all tested rootstocks were resistant (RF>1.00) to M. arenaria. In contrast, both tested grapevine scion cultivars were susceptible to the investigated Meloidogyne species. Index terms: occurrence, root-knot nematode, resistance, Vitis spp.
\end{abstract}

${ }^{1}$ (Trabalho 015-12). Recebido em: 03-01-2012. Aceito para publicação em: 10-08-2012.

${ }^{2}$ Bióloga, M. Sc. Doutoranda em Fitossanidade, Universidade Federal de Pelotas, Campus Universitário, S/N, 96001970, Capão do Leão-RS. E-mail: lsomavilla@hotmail.com

${ }^{3}$ Eng. Agr. Dr. Pesquisador da Embrapa Clima Temperado Pelotas, BR 392, Km 78, Caixa Postal 403, 96010-971, Pelotas-RS, E--mail: cbauer@cpact.embrapa.br

${ }^{4}$ Eng. Agr. Ph.D. Pesquisadora da Embrapa Uva e Vinho, R. Livramento 515, Caixa Postal 130, 95700-000, Bento Gonçalves-RS, E-mail: vera@cnpuv.embrapa.br 


\section{INTRODUÇÃO}

A viticultura brasileira ocupa uma área de aproximadamente 90 mil ha e apresenta produção em torno de um milhão e trezentas mil toneladas (IBGE, 2010), concentrando-se principalmente nas regiões Sul, Sudeste e Nordeste do Brasil. Problemas de ordem fitossanitária são responsáveis por sérios prejuízos à cultura da videira. Dentre esses, danos decorrentes do ataque de fitonematoides podem afetar o desenvolvimento e o estabelecimento das plantas no vinhedo, a qualidade dos frutos, a produção e os custos despendidos na cultura. Segundo Sasser (1989), perdas médias anuais, em decorrência do ataque da videira por fitonematoides, são estimadas em 12,5\%, entretanto a redução na produção pode chegar a 20\% (RASKI; KRUSBERG, 1984), o que caracteriza estes patógenos como um fator limitante à cultura (NAVES, 2005).

O gênero Meloidogyne (nematoide das galhas) é considerado um dos mais importantes causadores de danos em videiras (SOMAVILLA, 2011). Esse nematoide induz a formação de galhas nas raízes, restringindo a absorção de água, nutrientes e o crescimento da planta, além de poder predispôla ao ataque de outros patógenos. As espécies mais importantes desse gênero para a videira são $M$. incognita (Kofoide \& White) Chitwood, M. javanica (Treub) Chitwood, $M$. arenaria (Neal) Chitwood, $M$. hapla Chitwood e M. ethiopica Whitehead, sendo as quatro primeiras amplamente distribuídas na maioria das regiões onde a videira é cultivada (ANAWAR; MCKENRY, 2000; MOREIRA; LIMA, 2002; LORDELLO; LORDELLO, 2003). No entanto, essa última espécie de nematoide, apesar de ser relatada em videira somente no Chile, pode ocorrer em outros hospedeiros em países latino-americanos, como o Brasil, assim como na África e na Europa (CARNEIRO et al., 2003; SIRCA et al., 2004). A incidência de espécies de Meloidogyne nos vinhedos chilenos pode ser vista nos parreirais da região metropolitana de Santiago, onde se observa que a cada 5ha de videira, 1ha está contaminado com o nematoide (ABALLAY et al.,1997).

De acordo com informações acumuladas nos últimos 30 anos, na Califórnia-EUA, estimamse perdas no cultivo de uvas causadas por espécies de Meloidogyne, em torno de 20\%. Já na Flórida as perdas chegaram a 10\% (LORDELLO; LORDELLO, 2003). Porém, no Brasil, apesar de existirem relatos de danos decorrentes do ataque de Meloidogyne sp., não há dados de perdas por esse patossistema.

As estratégias usadas no manejo de nematoides na videira dependem da fase em que se encontra o empreendimento vitivinícola, podendo ser adotadas antes do plantio, em vinhedos já estabelecidos ou na renovação do vinhedo. $\mathrm{O}$ uso de porta-enxerto resistente ou tolerante ao nematoide das galhas é um método eficiente e econômico no controle dessa praga. Entretanto, a carência de informações relacionada a danos na videira e a reação de porta-enxertos às diferentes espécies de Meloidogyne que afetam a cultura, em nossas condições (SOMAVILLA, 2011), limitam o uso da resistência genética como estratégia de controle no manejo do nematoide das galhas.

Portanto, foi objetivo deste trabalho, relatar a ocorrência de $M$. incognita em videira no município de Petrolina-PE, e avaliar a reação de porta-enxertos e cultivares de copa de videiras a $M$. incognita e $M$. arenaria.

\section{MATERIAL E MÉTODOS}

\section{Relato e identificação do nematoide das galhas em videira}

Encontraram-se videiras (Vitis vinifera L.) da cultivar Festival (Superior Seedless) com a parte aérea mal desenvolvida, com menor número de folhas e folhagem mais esparsa, distribuídas em reboleiras, em um vinhedo situado na localidade da Serra do Urubu, município de Petrolina-PE, região do Vale do São Francisco. O sistema radicular era composto pelo porta-enxerto 'IAC 766-Campinas' ('106-8' x Vitis caribaea) onde se observou a presença de grande número de galhas associadas.

Nesse mesmo pomar, plantas de feijão-deporco (Canavalia ensiformis (L.) R. Wilcz), utilizadas como cobertura verde, também apresentavam o sistema radicular altamente atacado pelo nematoide das galhas.

A seguir, amostras de solo e de raízes de videira e de feijão-de-porco foram coletadas na reboleira, da qual foram obtidas amostras compostas de aproximadamente 100 g de raízes de videira e de feijão-de-porco e de $1 \mathrm{~kg}$ de solo. A seguir, as amostras foram devidamente identificada, acondicionadas em embalagem plástica e encaminhadas ao laboratório de nematologia da Embrapa Clima Temperado, Pelotas-RS, para análise nematológica. Posteriormente, as amostras de solo foram processadas em laboratório para a identificação e determinação do número de nematoides $/ 100 \mathrm{~cm}^{3} \mathrm{de}$ solo (JENKINS, 1964), e as raízes, dissecadas para extração das fêmeas e utilização na identificação da espécie do nematoide. Para tanto, fêmeas de Meloidogyne sp., de coloração branco-leitosa, foram extraídas de cada espécie vegetal, separadamente, 
e, imediatamente, submetidas à eletroforese para a identificação da(s) espécie(s) pelo polimorfismo das bandas esterásticas em gel de poliacrilamida a $6 \%$ (CARNEIRO; ALMEIDA, 2001).

Reação de porta-enxertos de videira a $M$. incognita e M. arenaria

Quatro porta-enxertos de videira tropicais ['IAC 313-Tropical' ('Golia' x Vitis cinerea), 'IAC 766-Campinas'(106-8 x V. caribaea), 'IAC 572-Jales' (V. caribaea e 101-14 Mgt), '106-8 Traviú' (V. riparia $\mathrm{x}(V$. rupestris $\mathrm{x} V$. cordifolia $))]$, oito porta-enxertos de clima temperado ['Solferino' $(V$. berlandieri $\mathrm{x}$ $V$. riparia), '420 A' ( $V$. berlandieri $\times$ V. riparia), 'Rupestris du Lot' (V. rupestris), 'SO4' (V. berlandieri $\mathrm{x} V$. riparia), 'K5BB Kober' ( $V$. berlandieri x $V$. riparia), '1103 Paulsen' (V. berlandieri $x$ V. rupestris), 'Harmony' (V. champinii x1613C) e 'Salt Creeck' ( $V$. candicans $\times \quad V$. rupestris)] e as cultivares copa 'Niágara Rosada'e ‘Chardonnay', propagados a partir de cultivo in vitro, foram avaliadas quanto à reação a $M$. incognita e $M$. arenaria, em condições de casa de vegetação, à temperatura de $25 \pm 5^{\circ} \mathrm{C}$.

Mudas individualizadas dos diferentes genótipos, mantidas em vasos com $2 \mathrm{~kg}$ de solo esterilizado, foram inoculadas com 10.000 ovos + J2 (juvenis de segundo estádio) de uma população pura de cada espécie de Meloidogyne (HUSSEY; BARKER, 1973), provenientes de videira e mantidas em tomateiro (Solanum lycopersicum L.) 'Santa Cruz', em casa de vegetação. O ensaio foi conduzido em delineamento completamente casualizado e constou de seis repetições para cada genótipo. Tomateiros cv. Santa Cruz também foram inoculadas com o mesmo nível de inóculo do nematoide, para averiguação de sua viabilidade.

Decorridos oito meses da inoculação, as raízes de cada planta foram separadas da parte aérea, lavadas e avaliadas quanto ao número de galhas. A seguir, realizou-se a extração de ovos das raízes (HUSSEY; BARKER, 1973) para a contagem do número de ovos por sistema radicular e posterior determinação do fator de reprodução (FR) de cada espécie de Meloidogyne (FR = população final/ população inicial) nos diferentes materiais genéticos testados. Consideraram-se como imunes os genótipos que apresentaram $\mathrm{FR}=0,00$; resistentes, $\mathrm{FR}<1,00$; e, suscetíveis, FR >1,00 (COOK; EVANS, 1987). Logo após, os valores para número de galhas (transformado em $\sqrt{x+1}$ ) e de FR de Meloidogyne spp., determinados para cada genótipo, foram submetidos à ANOVA, sendo as médias de cada tratamento comparadas entre si, pelo teste de agrupamento Scott-Knott, a 5\% de probabilidade.

\section{RESULTADOS}

\section{Identificação e quantificação do nematoide das galhas em videira}

Por meio das análises de eletroforese, identificou-se $M$. incognita com o fenótipo de esterase I2 (Rm: 1,00, 1,05), tanto nas amostras de raízes do porta-enxertos de videira 'IAC 766Campinas’, como nas raízes de feijão de porco.

Detectou-se a ocorrência de populações elevadas de $M$. incognita tanto nas amostras de solo provenientes de videira (717 J2 M. incognita $/ 100 \mathrm{~cm}^{3}$ solo) como nas de feijão-de-porco (26.720 J2 M. incognita $/ 100 \mathrm{~cm}^{3}$ solo). Além disso, também foi verificada a ocorrência de machos do nematoide das galhas nas amostras de solo, em ambos os hospedeiros, o que demonstra o alto nível de estresse destas plantas.

\section{Reação de porta-enxertos de videira a $M$. incognita e M. arenaria}

Os genótipos estudados demonstraram reação diferenciada quanto ao número de galhas e suscetibilidade às duas espécies de Meloidogyne. Analisando-se a variável número de galhas, os maiores valores foram detectados nas raízes das cultivares copa Chardonnay e 'Niágara Rosada' e do porta-enxerto '106-8 Traviú', independentemente da espécie de Meloidogyne inoculada (Tabela 1).

Quando avaliada a resistência dos materiais pelos valores de $\mathrm{FR}$, verificou-se que entre os porta-enxertos tropicais, 'IAC 313-Tropical' e 'IAC 572-Jales', foram resistentes a $M$. arenaria e M. incógnita, e o porta-enxerto '106-8 Traviú' foi suscetível às duas espécies do nematoide. No entanto, o porta-enxerto tropical 'IAC 766', apesar de resistente a $M$. arenaria, foi suscetível a $M$. incognita.

Os porta-enxertos de clima temperado 'Rupestris du Lot' e '420 A' foram suscetíveis às duas espécies de Meloidogyne; no entanto, '1103 Paulsen’ apresentou resistência a $M$. incognita e suscetibilidade a $M$. arenaria. Os demais portaenxertos de clima temperado apresentaram reação de resistência para ambas espécies de Meloidogyne testadas. A reação de imunidade foi constatada apenas no porta-enxerto 'Solferino' a M. incognita, o qual também se comportou como resistente a $M$. arenaria (Tabela 1$)$. 


\section{DISCUSSÃO}

Os elevados níveis de $M$. incognita no solo e a presença de machos nas amostras demonstram o alto nível de estresse dessas plantas frente aos sintomas observados a campo. Além do mais, o cultivo de feijão-de-porco como cobertura verde no pomar pode ter favorecido o aumento do nível populacional do nematoide no solo por ser uma cultura suscetível a $M$. incognita. Dessa forma, o uso desta leguminosa, muito provavelmente, pode ter contribuído para o declínio da videira, conforme demonstrado pela ocorrência dos sintomas de amarelecimento e redução da área foliar relatados anteriormente.

No Brasil, a ocorrência de $M$. incognita já foi relatada em vinhedos nos municípios de Andradas, Caldas e Santa Rita de Caldas, no Estado de Minas Gerais, em Pelotas-RS, Tunápolis-SC e Verê-PR (GOMES, 2003). Assim, a identificação correta da espécie do nematoide é de relevante importância no manejo dessa praga, em uma área infestada, seja pela adoção de um porta-enxerto resistente, seja pelo uso de culturas não hospedeiras que possam ser cultivadas em consórcio ou empregadas como cobertura verde.

Dentre os porta-enxertos tropicais testados, 'IAC 766-Campinas' e '106-Traviú' foram suscetíveis a $M$. incognita. Na literatura, não há informações disponíveis sobre a reação de resistência desses materiais a Meloidogyne spp., exceto em um relato de Camargo (1998), onde 'IAC 313-Tropical' foi referido como tolerante a nematoides. Porém, aquele autor não informou quais nematoides e/ou espécies estão relacionados a este genótipo. Desta forma, os sintomas observados em videira e relacionados ao ataque de $M$. incognita, a campo, corroboram a reação de suscetibilidade de 'IAC 766-Campinas' ao nematoide, verificada posteriormente nesse mesmo porta-enxerto.

Apesar de os porta-enxertos de clima temperado 'Rupestris du Lot' e '420A' terem-se comportado como suscetíveis a $M$. incognita e $M$. arenaria, os mesmos foram descritos na literatura como resistente (NOGUEIRA, 1984) ou tolerante (URIZ, 1986) a ambas as espécies de Meloidogyne. Da mesma forma, apesar de o porta-enxerto '1103 Paulsen' ter se comportado como suscetível a $M$. arenaria e, porém, resistente a $M$. incognita, em outros trabalhos, esse mesmo genótipo é considerado moderadamente resistente ou suscetível a $M$. incognita e resistente a $M$. arenaria (BOULBAS, 1992). De acordo com Loubser e Meyer (1987), as diferenças observadas são atribuídas ao grau de resistência de 'Paulsen 1103’ a Meloidogyne spp., o qual pode ser modificada por condições de campo como fertilidade do solo, irrigação, diferenças clonais do porta-enxerto bem como pela variação na virulência da espécie do nematoide.

Os porta-enxertos de clima temperado 'Salt Creek', 'K5BB', 'SO4', 'Harmony’ e 'Solferino' apresentaram resistência a ambas as espécies testadas. Com exceção dos dois últimos porta-enxertos, os demais foram referidos na literatura como resistentes às espécies de maior frequência no Brasil, $M$. incognita, M. javanica, M. arenaria e $M$. hapla (CAMPOS et al., 2003; TÉLIS; LANDA, 2007). Além do mais, 'SO4' foi resistente a Tylenchulus semipenatrans e Pratylenchus spp. (PINOCHET et al., 1982), 'Salt Creeck' apresentou boa resistência a Xiphinema index (COIRO et al., 1990) e é considerado moderadamente resistente à filoxera (Daktulospharia vitifoliae) (WOLPERT et AL., 1992).

Apesar da resistência de 'Harmony' a $M$. incognita e $M$. arenaria, este genótipo tem seu uso restrito por apresentar características culturais indesejáveis e ser de propagação difícil, o que poderia limitar seu uso na prática; porém, sua inclusão em programas de melhoramento de porta-enxertos de videira pode contribuir para a obtenção de materiais agronomicamente desejáveis e resistentes a Meloidogyne spp.. O fato de 'Solferino' ter apresentado imunidade a $M$. incognita e resistência a $M$. arenaria nesse estudo, em contraponto aos resultados de Uris (1986), que o considerou altamente suscetível a M. incognita, M. javanica e M. arenaria, também pode ser atribuído a diferentes raças e protótipos de espécies de Meloidogyne, conforme relatado anteriormente.

A suscetibilidade das cultivares copa Niágara Rosada e Chardonnay a $M$. incognita e $M$. arenaria reforça a importância do uso de porta-enxertos resistentes como estratégia de controle onde esses nematoides ocorrem. Tais evidências somam-se aos resultados obtidos por Gomes et al. (2009) em levantamento nematológico realizado na Serra Gaúcha, onde os autores verificaram a ocorrência de $M$. arenaria em mais de $36 \%$ dos pomares de videira formados diretamente por plantas obtidas de pés-francos das cultivares de videira Niágara Rosada e Concord.

O uso de porta-enxertos resistente é a medida mais eficiente para controle de fitonematoides, pois além de evitar o aumento das populações desses patógenos, atende ao interesse do produtor em obter maior produção, melhor qualidade dos frutos e menores custos de produção. No entanto, o uso de porta-enxertos de videiras resistentes a Meloidogyne 
spp. em associação com outras características, como resistência à filoxera, à pérola-da-terra (Eurhizococcus brasiliensis Hempel), aos fungos de solo ou tolerância ao encharcamento do solo, pode constituir uma ferramenta fundamental no manejo integrado de pragas de importância agrícola para a videira.

TABELA 1 - Reação de genótipos de videira a Meloidogyne incognita e M. arenaria, Pelotas-RS.

\begin{tabular}{lrrrrrr}
\hline \multirow{2}{*}{ Genótipos } & \multicolumn{3}{c}{ Meloidogyne incognita } & \multicolumn{3}{c}{ Meloidogyne arenaria } \\
\cline { 2 - 7 } & \multicolumn{1}{c}{$\begin{array}{c}\mathbf{N}^{0} \text { de } \\
\text { Galhas }\end{array}$} & \multicolumn{1}{c}{ FR } & Reação & $\begin{array}{r}\text { N }{ }^{0} \text { de } \\
\text { Galhas }\end{array}$ & FR & Reação \\
\hline Tomateiro Santa Cruz'1 & $902,83 \mathrm{a}$ & $32,37 \mathrm{a}$ & $\mathrm{S}$ & $483,5 \mathrm{c}$ & $26,11 \mathrm{a}$ & $\mathrm{S}$ \\
'Chardonnay' & $398,80 \mathrm{~b}$ & $13,36 \mathrm{~b}$ & $\mathrm{~S}$ & $1362,00 \mathrm{a}$ & $16,03 \mathrm{~b}$ & $\mathrm{~S}$ \\
'106-8 Traviú' & $325,44 \mathrm{~b}$ & $11,09 \mathrm{c}$ & $\mathrm{S}$ & $477,80 \mathrm{c}$ & $7,46 \mathrm{c}$ & $\mathrm{S}$ \\
'IAC 766-Campinas' & $91,26 \mathrm{~d}$ & $8,67 \mathrm{~d}$ & $\mathrm{~S}$ & $0,00 \mathrm{f}$ & $0,03 \mathrm{f}$ & $\mathrm{R}$ \\
'Rupestris du Lot' & $245,66 \mathrm{c}$ & $7,03 \mathrm{e}$ & $\mathrm{S}$ & $340,50 \mathrm{~d}$ & $6,27 \mathrm{c}$ & $\mathrm{S}$ \\
'Niágara Rosada' & $336,00 \mathrm{~b}$ & $6,85 \mathrm{e}$ & $\mathrm{S}$ & $627,30 \mathrm{~b}$ & $4,78 \mathrm{~d}$ & $\mathrm{~S}$ \\
'420 A' & $1,16 \mathrm{f}$ & $1,15 \mathrm{f}$ & $\mathrm{S}$ & $133,80 \mathrm{e}$ & $2,29 \mathrm{e}$ & $\mathrm{S}$ \\
'Harmony' & $0,00 \mathrm{f}$ & $0,10 \mathrm{~g}$ & $\mathrm{R}$ & $0,00 \mathrm{f}$ & $0,20 \mathrm{f}$ & $\mathrm{R}$ \\
'Salt Creek' & $43,16 \mathrm{e}$ & $0,03 \mathrm{~g}$ & $\mathrm{R}$ & $16,10 \mathrm{f}$ & $0,21 \mathrm{f}$ & $\mathrm{R}$ \\
'1103 Paulsen' & $0,00 \mathrm{f}$ & $0,03 \mathrm{~g}$ & $\mathrm{R}$ & $283,30 \mathrm{~d}$ & $5,00 \mathrm{~d}$ & $\mathrm{~S}$ \\
'IAC 572-Jales' & $0,00 \mathrm{f}$ & $0,02 \mathrm{~g}$ & $\mathrm{R}$ & $0,30 \mathrm{f}$ & $0,07 \mathrm{f}$ & $\mathrm{R}$ \\
'K5BB Kober' & $3,50 \mathrm{f}$ & $0,02 \mathrm{~g}$ & $\mathrm{R}$ & $7,10 \mathrm{f}$ & $0,40 \mathrm{f}$ & $\mathrm{R}$ \\
'IAC 313-Tropical' & $0,00 \mathrm{f}$ & $0,01 \mathrm{~g}$ & $\mathrm{R}$ & $1,10 \mathrm{f}$ & $0,48 \mathrm{f}$ & $\mathrm{R}$ \\
'SO4' & $0,50 \mathrm{f}$ & $0,01 \mathrm{~g}$ & $\mathrm{R}$ & $16,10 \mathrm{f}$ & $0.19 \mathrm{f}$ & $\mathrm{R}$ \\
'Solferino' & $0,00 \mathrm{f}$ & $0,00 \mathrm{~g}$ & $\mathrm{I}$ & $2,80 \mathrm{f}$ & $0,07 \mathrm{f}$ & $\mathrm{R}$ \\
\hline \multicolumn{1}{c}{ CV (\%) } & 44,4 & 18,57 & & 35,8 & 29,07 & \\
\hline
\end{tabular}

${ }^{1}$ Testemunha; FR - Fator de Reprodução; *Médias seguidas pela mesma letra, na coluna, não diferem entre si, pelo teste de Scott-knott, $5 \%$ ** Valores originais transformados em $\sqrt{x+1}$ (S) suscetível; (R) resistente; (I) Imune; CV= Coeficiente de variação

\section{CONCLUSÃO}

O uso de porta-enxertos resistente é a medida mais eficiente para controle de fitonematoides, pois além de evitar o aumento das populações desses patógenos, atende ao interesse do produtor em obter maior produção, melhor qualidade dos frutos e menores custos de produção. No entanto, o uso de porta-enxertos de videiras resistentes a Meloidogyne spp. em associação com outras características, como resistência à filoxera, à pérola-da-terra (Eurhizococcus brasiliensis Hempel), aos fungos de solo ou tolerância ao encharcamento do solo, pode constituir uma ferramenta fundamental no manejo integrado de pragas de importância agrícola para a videira.

\section{REFERÊNCIAS}

ABALLAY, E.: BAETTIG, R. Y.: VIEIRA, A. Evaluación de la tolerancia de ocho portainjertos de vid al nemátodo del nódulo de la raíz (Meloidogyne spp.). Aconex, Santiago, n.56, p.15-21, 1997.

ANAWAR, S. A.; McKENRY, M. V. Penetration, development and reproduction of Meloidogyne arenaria on two new resistant Vitis spp. Nematropica, Bradenton, v.30, n.1, p.9-17, 2000.

BOUBALS, D. Au sujet de la résistance à l'anguillule de la vigne. Le Progrès Agricole et Viticole, Année, v.109, n.2, p.118, 1992.

CAMARGO, U. A. Cultivares para a viticultura tropical do Brasil. Informe Agropecuário, Belo Horizonte, v.19, n.194, p.15-19, 1998. 
CAMPOS, V. P.; MAXIMINIANO, C.; FERREIRA, E. A. Uva para processamento: fitossanidade. Bento Gonçalves: Embrapa Uva e Vinho, Brasília: EMBRAPA, 2003. (Frutas do Brasil).

CARNEIRO, R. M. D. G.; ALMEIDA, M. R. A. Técnica de eletroforese usada no estudo de enzimas dos nematoides das galhas para identificação de espécies. Nematologia Brasileira, Brasília, v.25, n.1, p.35-44, 2001.

CARNEIRO, R. M. D. G,; GOMES, C. B.; ALMEIDA, M. R.; GOMES, A. C. C.; MARTINS, I, Primeiro registro de Meloidogyne ethiopica Whitehed, 1968 em plantas quivi no Brasil e reação em diferentes plantas hospedeiras. Rev. Nematologia Brasileira, Brasilia, v.27, n.2, p. 152-158, 2003.

COOK, C.G.; EVANS, K. Resistance and tolerance. In: BROWN, R.H.; KERRY, B.R. (Ed.). Principles and practice of nematode control in crops. Orlando: Academic Press, 1987. p.179-231.

COIRO, M. I.; TAYLOR C.E.; BORGO M.; LAMBERTI F. Resistance of grapevine rootstocks to Xiphinema index. Nematologia Mediterranea, Bari, v.18, n.2, p.119-121, 1990.

GOMES, C. B. Problemas nematológicos associados a videira. In: CONGRESSO BRASILEIRO DE NEMATOLOGIA, 24., 2003. Anais... p.26-30.

GOMES, C. B.; CAMPOS, A. D.; COSTA, F.A. Levantamento de nematoides fitoparasitas associados a pomares de videira em declínio da Serra Gaúcha. Pelotas: Embrapa Clima Temperado, 2009. (Boletim de Pesquisa e Desenvolvimento, 110).

HUSSEY, R. S.; BARKER, K. B. A comparison of methods of collecting inocula for Meloidogyne spp., including a new technique. Plant Disease, Saint Paul, v.57, n.7, p.1025-1028, 1973.

JENKINS, W. R. A rapid centrifugal flotation technique for separating nematodes from soil. Plant Disease Reporter, St Paul, v.48, n.9, p.692-695, 1964.

LORDELLO, R. R. A.; LORDELLO, A. I. L. Doenças e nematoides In: POMER, C.V. Uva: tecnologia de produção, pós-colheita, mercado. Porto Alegre: Cinco Continentes, 2003. p.568-596.
LOUBSER., J. T.; MEYER., A. J. Resistance of Grapevine rootstocks to Meloidogyne incognita under field conditions. South African Journal of Enology and Viticulture, South Africa, v.2, n.8, p.70-74, 1987.

MOREIRA, W. A.; LIMA, M. F. Uva de mesa: fitossanidade. Petrolina: Embrapa Semi-Árido, 2002. p.45-52. (Frutas do Brasil).

NAVES, R. L Diagnose e manejo de doenças causadas por fitonematoides na cultura da videira. Bento Gonçalves: Embrapa uva e vinho, 2005. (Circular Técnica)

PINOCHET, J.; VERDEJO, S.; SOLER, A.; CANAL, J. Host range of a population of Pratylenchus vulnus in comercial fruit, nut, citrus, and grape rootstocks in Spain. Journal of Nematology, College Park, v.24, n.4, p.51-54, 1982.

NOGUEIRA, D. J. P. Porta-enxerto de videira. Informe Agropecuário, Belo Horizonte, v.10, n.117, p.22-24, 1984

RASKI, D. J.; KRUSBERG, L. R. Nematode parasites of grapes and other smail fruits. In: NICKLE, W. R. (Ed.). Plant and insect nematodes. New York: Marcel Dekker, 1984. cap.13, p.457-507.

SASSER, J. N. Plant parasitic nematodes: the farmer's hidden enemy. Raleigh: Departament of Plant Pathology and Consortium of International Crop Protection, North Caroline State University, 1989. 115p.

SIRCA, S.; UREK. G.; KARSSEN, G. First report of the roo-knot nematode Meloidogyne ethiopica in Europe. Plant Disease, St Paul, v.88, n.6, p.680, 2004.

SOMAVILLA, L. Levantamento, caracterização do nematoide das galhas em videira nos estados do rio grande do sul e de santa catarina e estudo da resistência de porta-enxertos a Meloidogyne spp. 2010. 81f. Tese (Doutorado em Fitossanidade) - Faculdade de Agronomia Eliseu Maciel, Universidade Federal de Pelotas, Pelotas, 2011.

TÉLIS, D.; LANDA, B. B. Plant-parasitic nematodes infecting grapevine in Southern Spain and susceptible reaction to root-knot nematodes of rootstocks reported as moderately resistant. Plant Disease, St Paul, v.91, n.9, p.1147-1154, 2007. 
URIS, J. O. Nematodes. In: España. Ministerio de Agricultura, Pesca y Alimentación. Dirección general de la produción agrária. Subdirección General de Sanidad Vegetal. Los parasitos de la vid: estrategia de lucho. Madri, 1986. p.125-128.
WOLPERT, J.A.; WALKER, M.A.; WEBER, E. Proceedings roostock seminar: a worldwide perspective. Reno: The American society for Enology and Viticulture, 1992. 84p. 